\title{
LeGes
}

Lucas Stutz

\section{Deutschschweizerische Gesetzessprache - die kantonalen Richtlinien im Vergleich}

Dieser Beitrag vergleicht die gesetzestechnischen Richtlinien der deutschsprachigen Kantone hinsichtlich ihrer Empfehlungen zur Gesetzessprache. Zunächst werden die allgemeinen Anforderungen an die Gesetzessprache diskutiert, allen voran die Knappheit und Allgemeinverständlichkeit. Dann wird mit der Wortwahl und dem Satzbau auf zwei weitere zentrale Aspekte der Gesetzessprache eingegangen. Dabei werden die Empfehlungen nicht nur verglichen, sondern auch unter Einbezug von wissenschaftlicher Literatur kritisch diskutiert. Der Vergleich zeigt, dass die Kantone zwar dieselben Ansprüche an die Gesetzessprache stellen, dass sie jedoch teilweise unterschiedliche Ansätze verfolgen, diese umzusetzen, oder überhaupt keine Lösungsvorschläge anbieten.

Beitragsart: Wissenschaftliche Beiträge

Zitiervorschlag: Lucas Stutz, Deutschschweizerische Gesetzessprache - die kantonalen Richtlinien im Vergleich, in: LeGes 32 (2021) 2 


\section{Inhaltsübersicht}

1. Einleitung

2. Allgemeine Anforderungen an die Gesetzessprache

2.1. Knappheit

2.2. Allgemein- vs. Fachsprachlichkeit

3. Wortwahl

3.1. Lexikalische Kohärenz

3.2. Textsortenspezifische Terminologie

3.3. Legaldefinitionen

4. Satzbau: Informationsdichte

4.1. Ellipsen

4.2. Nominalstil

4.3. Multipropositionale Sätze

5. Satzbau: Sprachliche Komplexität

5.1. Sinnvolle und weniger sinnvolle Änderungen

5.2. Die Causa Passivsatz

5.3. Perspektive

6. Zusammenfassung und Fazit

\section{Einleitung}

[1] Als im April 2019 die vierte Auflage des Gesetzgebungsleitfadens des Bundesamtes für Justiz publiziert wurde, suchte man darin vergebens nach dem Kapitel über die Gesetzessprache. Dieses ist mit der Begründung weggelassen worden, dass es zu schwierig sei, Kohärenz zwischen der deutschen und französischen Sprachfassung herzustellen (Bundesamt für Justiz 2019: 3). Obwohl das besagte Kapitel unterdessen auf die Website der Sprachdienste der Bundeskanzlei «hinübergerettet» worden ist und digital zur Verfügung steht ${ }^{1}$, bleibt im Gesetzgebungsleitfaden bezüglich der Gesetzessprache eine Lücke zurück.

[2] Doch nicht nur der Bund setzt sich mit der Frage auseinander, was eine gute Gesetzessprache ausmacht. Auch zahlreiche Kantone thematisieren diese Frage in ihren legistischen Richtlini$e^{2}$. Das Zentrum für Rechtsetzungslehre der Universität Zürich hat diese Richtlinien gesammelt und stellt sie online zur Verfügung ${ }^{3}$. Die Sammlung wurde 2020 aktualisiert. Von den insgesamt 20 deutschsprachigen Richtlinien äussern sich 19 zur Gesetzessprache. Dabei reicht das Spektrum von eher knapp gehaltenen Anmerkungen ${ }^{4}$ über das Vertiefen der wichtigsten Punkte ${ }^{5}$ bis hin zu sehr umfangreichen Kommentaren mit Beispielen und Empfehlungen ${ }^{6}$.

[3] Ein Vergleich dieser Empfehlungen zur Gesetzessprache wurde bisher noch nicht vorgenommen. Zwar wurden gesetzestechnische Richtlinien schon vergleichend untersucht, so etwa bei Müller (1976) und KindermanN (1979), jedoch ohne besondere Berücksichtigung der Geset-

1 https://www.bk.admin.ch/bk/de/home/dokumentation/sprachen/hilfsmittel-textredaktion/gesetzessprache. html, Stand 16. Juni 2021.

2 Auf die einzelnen Richtlinien wird durch das Kantonskürzel mit Seitenangabe (getrennt durch einen Doppelpunkt) verwiesen. Im Falle von BE betrifft die Seitenzahl stets das Modul 4 (Sprache), sofern nichts anderes angegeben wird. Dem Vergleich wird die Zusammenstellung des Zentrums für Rechtsetzungslehre (Stand: 16. Juni 2021) zugrunde gelegt.

3 https://www.zfr.uzh.ch/de/dienstleistungen/richtlinien.html, Stand 16. Juni 2021.

4 SH:5, BS:15, UR:10f., AG:6, FR, LU:13, TG:14, VS:7, AR:7, GR:14, SZ:27, SO:6f.

5 AI:15, GL:18, OW:10ff.

6 ZG:32ff., SG:46, ZH:64ff., BE:1ff. 
zessprache. HöfLer (2012) geht zwar auf die Gesetzessprache ein, beschränkt sich aber auf die Richtlinien der Kantone Bern und Zürich. Die vorliegende Arbeit schliesst diese Lücke, indem sie spezifisch auf die Richtlinien der deutschsprachigen Kantone und auf die darin enthaltenen Empfehlungen zur Gesetzessprache fokussiert. Neben dem blossen Vergleich hat sich die Untersuchung auch eine kritische Betrachtung der Empfehlungen zum Ziel gesetzt. Mit Bezug auf die wissenschaftliche Literatur zum jeweiligen Thema soll nicht nur thematisiert werden, worin die Richtlinien divergieren, sondern es sollen auch allgemeine Grundsätze und Faustregeln hinterfragt werden.

[4] Dafür werden die gesetzestechnischen Richtlinien der deutschsprachigen Kantone auf drei Aspekte hin verglichen und diskutiert: die allgemeinen Anforderungen an die Gesetzessprache (Abschnitt 2), die Wortwahl (Abschnitt 3) und den Satzbau, wobei dort zwischen Informationsdichte (Abschnitt 4) und sprachlicher Komplexität (Abschnitt 5) unterschieden wird.

\section{Allgemeine Anforderungen an die Gesetzessprache}

[5] Die legistischen Richtlinien der Kantone stellen eine Reihe genereller Anforderungen an die Gesetzessprache, über die ein breiter Konsens zu herrschen scheint: Die Gesetzessprache soll einfach ${ }^{7}$, klar $^{8}$ und verständlich ${ }^{9}$ sein. Das Kriterium der Klarheit findet sich auch in Artikel 7 des Sprachgesetzes wieder, dessen Sachüberschrift zudem auf die Verständlichkeit verweist:

Art. 7 Verständlichkeit

Die Bundesbehörden bemühen sich um eine sachgerechte, klare und bürgerfreundliche Sprache und achten auf geschlechtergerechte Formulierungen. (Art. 7 Abs. 1 $\mathrm{SpG}^{10}$ )

[6] Des Weiteren wird häufig angeführt, dass Erlasse knapp und kurz ${ }^{11}$ sowie präzise ${ }^{12}$ sein müssen. Mit der Knappheit hängt der Anspruch zusammen, in Rechtssätzen dürfe nur Normatives stehen ${ }^{13}$, während in Bezug auf die Präzision die Frage nach dem Verhältnis zwischen Fach- und Allgemeinsprachlichkeit relevant ist. Diese beiden Anforderungen werden im Folgenden eingehender diskutiert.

\subsection{Knappheit}

[7] Die Forderung nach Knappheit in Erlasstexten scheint zwei unterschiedliche Forderungen zu beinhalten: Gemäss den Richtlinien kann sich die Knappheit auf den Text insgesamt ${ }^{14}$ oder ledig-

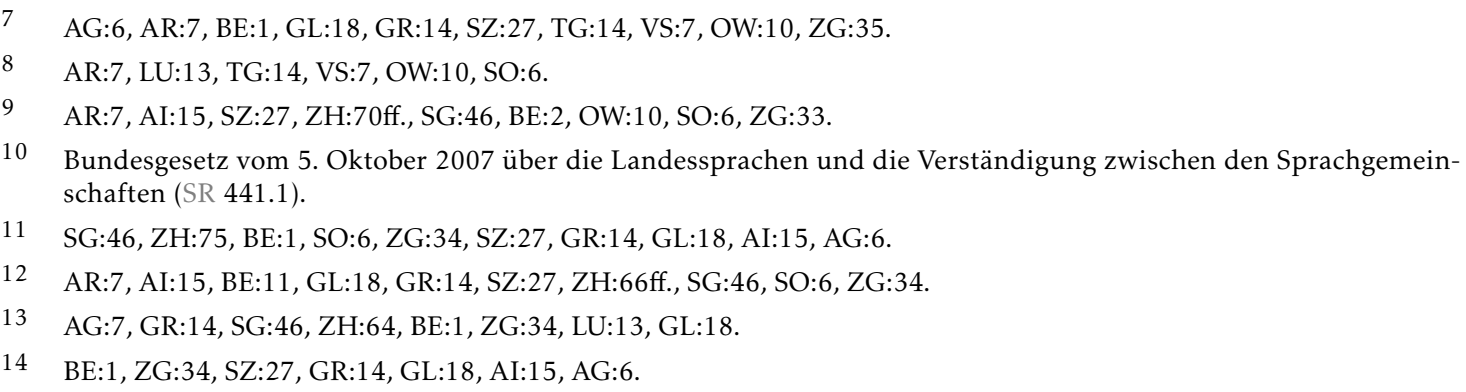


lich auf seine einzelnen Bestandteile wie Artikel oder Rechtssätze ${ }^{15}$ beziehen. Nicht immer geht aus den Richtlinien eindeutig hervor, ob beide dieser Aspekte oder nur einer davon gemeint ist (z. B. SG:46). Natürlich besteht ein Zusammenhang - je kürzer die einzelnen Sätze und Artikel sind, desto kürzer wird der Text insgesamt - doch die Umsetzung der Kürze wie auch deren Funktion ist nicht in beiden Fällen dieselbe. In diesem Abschnitt wird nur die Kürze der Gesamttexte thematisiert. Strategien zur Verkürzung einzelner Rechtssätze werden dagegen im Abschnitt zum Satzbau diskutiert.

[8] Als Herausforderung für die Umsetzung der Knappheit erweist sich der scheinbar gegenläufige Anspruch der Vollständigkeit ${ }^{16}$ : Je mehr Informationen ein Text beinhaltet, je mehr Differenzierungen, Ausnahmen etc. vorgenommen werden müssen, desto länger wird er. Die kantonalen Richtlinien bieten eine Reihe von Mitteln an, um auch einen informationsreichen Text möglichst kurz zu halten:

- Reduktion des Inhalts auf das Notwendigste (z. B. AI:16)

- Weglassen von Selbstverständlichkeiten (z. B. ZG:34, VS:6)

- nur Sätze mit normativer Relevanz (vgl. Fussnote 13)

- keine Wiederholungen (ZH:76, SG:46)

- Zusammenfassen und Verallgemeinern einzelner Normen, sofern inhaltlich möglich (ZH:76)

- logische Reduktion durch Klammertechnik (ZH:76)

- logische Reduktion durch geschicktes Verwenden von Ober- und Unterbegriffen (ZH:76)

- Verwenden von Legaldefinitionen (z. B. ZH:76, ZG:34)

- Verwenden von normativen Verweisungen (ZH:77)

[9] Der Grundgedanke hinter der Forderung nach Kürzung ist ein simpler: Je mehr gelesen und verarbeitet werden muss, desto grösser ist der Lektüreaufwand. Diese etwas banal anmutende Aussage lädt dazu ein, den Wert der Kürze eines Textes etwas kritischer zu betrachten. Es wäre falsch zu behaupten, dass längere Texte prinzipiell unverständlicher seien als kürzere. Immerhin sind weite Teile der Bevölkerung in der Lage, mehrere hundert Seiten starke Romane zu lesen.

[10] Ermöglicht wird dies durch ein mentales Modell des Textinhalts. Es handelt sich dabei um eine vereinfachte mentale Repräsentation sowohl des Inhalts wie auch der Funktion eines Textes (vgl. Richter/Schnotz 2018). Solche Textmodelle sind nicht nur eine «mentale Zusammenfassung», sondern helfen auch dabei, das neu Gelesene zu verstehen: Irrelevante Informationen können gefiltert und relevante Informationen können ins Modell integriert werden.

[11] Gesetzestexte mit Unterhaltungsliteratur zu vergleichen ist jedoch problematisch. Abgesehen von den unterschiedlichen Anwendungsbereichen unterscheiden sich diese Textsorten auch in der Art und Weise der Lektüre: Erlasstexte werden üblicherweise punktuell konsultiert (vgl. Nussbaumer et al. 1995: 96; Nussbaumer 1995: 100f.). Das mentale Modell eines Erlasstextes besteht weniger darin, alle Normen eines bestimmten Erlasses auswendig zu kennen - das wäre eine immense kognitive Leistung, welche auch die beste Textgestaltung kaum ermöglichen

15 ZH:76, BE:4, SO:6, ZG:34, SZ:27, GR:14, GL:18, AI:16, AG:6.

16 AI:15, SG: 46, ZG:67. 
könnte - sondern darin, die benötigten Normen aufzufinden. Dass diese Leistung nicht durch die Kürze des Textes, sondern durch dessen Struktur ermöglicht wird, zeigt der Vergleich mit einem Lexikon: Ein Lexikon ist nicht schwerer zu verwenden, wenn es statt tausend zweitausend Seiten hat. Dagegen wäre es völlig unbrauchbar, wenn die einzelnen Artikel nicht alphabetisch, sondern in zufälliger Reihenfolge angeordnet wären.

[12] Wichtig ist jedoch, dass die einzelnen Artikel in sich nicht zu komplex sind - dies gilt sowohl für Lexika wie auch für Erlasstexte. In diesem Zusammenhang erwähnen einige kantonale Richtlinien ${ }^{17}$ die sogenannte Eugen-Huber-Regel. Diese geht auf den gleichnamigen Schweizer und Verfasser des Schweizerischen Zivilgesetzbuches zurück und nimmt ebenfalls auf die Länge einzelner Textelemente (und nicht auf die Länge des Gesamttextes) Bezug:

1. pro Artikel höchstens drei Absätze

2. pro Absatz ein Satz

3. pro Satz ein Gedanke

[13] Aber auch verknappte Sätze sind nicht immer vorteilhaft. Lassen Verfasserinnen und Verfasser gewisse Informationen aus, die sie als selbstverständlich oder überflüssig erachten, so kann für die Leserinnen und Leser ein kognitiver Mehraufwand entstehen, da nicht Gesagtes ergänzt werden muss (Hartmann/Parádi 2018: 217f.). Die Vertrautheit mit einem Text und die damit einhergehenden «mentalen Abkürzungen» können bei den Leserinnen und Lesern nicht ohne Weiteres vorausgesetzt werden (vgl. ebd.: 233-236). In solchen Fällen ist es durchaus angebracht, dem Text Informationen hinzuzufügen, die streng logisch gesehen nicht unbedingt nötig wären (vgl. ebd.: 217f.).

[14] Das folgende Beispiel (entnommen aus Hartmann/Parádi 2018: 233) zeigt die möglichen Nachteile einer zu knappen Formulierung von Rechtssätzen auf:

Der Prüfling soll praxisbezogene Aufgaben schriftlich bearbeiten.

[15] Für sich alleine betrachtet, scheint dieser Rechtssatz zwei Normen auszudrücken: Er legt erstens fest, dass Prüflinge ihre Aufgaben schriftlich bearbeiten müssen, und zweitens, dass diese Aufgaben praxisbezogen sein müssen. Dabei wird aber offengelassen, ob grundsätzlich alle dieser Aufgaben praxisorientiert sind oder ob es auch Ausnahmen gibt; respektive ob die schriftliche Bearbeitung nur für die eine Kategorie von Aufgaben gilt, nicht jedoch für die andere.

[16] Um solche Unklarheiten zu vermeiden, lohnt es sich, eine längere Formulierung in Kauf zu nehmen, auch wenn dafür mehrere Sätze nötig sind. So könnten beispielsweise die beiden Normen im oberen Beispiel wie folgt in separaten Sätzen ausformuliert werden:

Der Prüfling soll die Aufgaben schriftlich bearbeiten.

Die Aufgaben müssen praxisbezogen sein.

[17] Zusammenfassend kann über die Knappheit gesagt werden, dass sie für die Verständlichkeit einzelner Artikel und Sätze relevant ist, nicht aber für den gesamten Erlasstext. In Erlasstexten nämlich kommt dem Aufbau eine wichtigere Rolle zu. Ein sinnvoller Textaufbau kann die Span-

17 BE:2, VS:7, AG:6, ZG:34, SO:8. 
nung zwischen Kürze und Vollständigkeit bis zu einem gewissen Grad aufheben, da er das Auffinden der benötigten Norm vereinfacht - auch in einem längeren Text. Bei Textkürzungen muss zudem stets die Vertrautheit der Leserinnen und Leser mit der Materie berücksichtigt werden, die üblicherweise geringer ist als diejenige der Verfasserinnen und Verfasser.

\subsection{Allgemein- vs. Fachsprachlichkeit}

[18] Im Zusammenhang mit der Verständlichkeit von Gesetzestexten stellt sich immer wieder die Frage, ob Formulierungen eher fach- oder eher allgemeinsprachlich ausgerichtet sein sollten. Diesbezüglich beziehen die kantonalen Richtlinien verschiedene Positionen:

1. Erlasstexte müssen allgemeinverständlich und somit auch für juristische Laiinnen und Laien verständlich sein (AI:15, SZ:27, VS:7, TG:14).

2. Erlasstexte müssen grundsätzlich allgemeinverständlich sein, allerdings darf bei den Leserinnen und Lesern ein gewisses Vorwissen vorausgesetzt werden. Spezielle Begriffe können mit Legaldefinitionen eingeführt werden (GL:18, ZG:33, SG:49, ZH:70).

3. Die Allgemeinsprachlichkeit ist abhängig vom Adressatenkreis: Betreffen die Normen einen grossen Teil der Rechtsunterworfenen, so ist es für die Funktionalität des Erlasses notwendig, dass er auch von der breiten Bevölkerung verstanden werden kann. Handelt es sich dagegen um hochgradig spezialisierte Regelungsgegenstände, die nur eine bestimme Gruppe von Fachleuten betreffen, ist die Fachsprache angebracht (AG:6, OW:10, LU:13). Die verwendete Sprache muss den Adressaten angepasst werden (BE:2). Fachausdrücke dürfen nicht zugunsten begrifflicher Klarheit vereinfacht werden (VS:7).

4. Erlasstexte müssen nicht allgemeinverständlich sein, da sich Laiinnen und Laien üblicherweise über andere Kanäle als den Erlasstext über die geltenden Normen informieren. Im Vordergrund steht deshalb die Vermittelbarkeit von Erlasstexten (GR:14).

[19] Die Mehrheit der Richtlinien plädiert also für eine zumindest prinzipielle Allgemeinverständlichkeit. Dafür sprechen auch Demokratie, Rechtsstaatlichkeit und die angestrebte Wirksamkeit des Rechts (vgl. HöFLER 2018: 67-72). So unterstehen beispielsweise viele Erlasse dem Referendum, weshalb die Rechtsunterworfenen eine «faire Chance haben [müssen], die einschlägigen Erlasse ohne fremde Hilfe verstehen zu können» (ZG:33), selbst wenn sie nicht direkt von diesen Erlassen betroffen sind. Um dieses Ziel umzusetzen, gelte es, «Amtsdeutsch» (LU:13, BE:3) und allgemein den «Fachjargon» (LU:13) zu vermeiden.

[20] Auch in der Bevölkerung scheint ein Bedürfnis nach Allgemeinverständlichkeit von Gesetzen zu bestehen, wie eine Studie der Gesellschaft für deutsche Sprache nahelegt (ЕіснноғF-Cyrus et al. 2009). Dieser Befund muss allerdings in zweierlei Hinsicht relativiert werden: Erstens handelt es sich um eine Befragung, die in den Nullerjahren in Deutschland durchgeführt worden ist, und zweitens betrifft sie nicht nur Gesetze, sondern die Behördenkommunikation im Allgemeinen. Während man annehmen kann, dass sich die Meinungen der Schweizerinnen und Schweizer nicht frappant von denen deutscher Bürgerinnen und Bürgern unterscheiden, darf man nicht vergessen, dass sich die Durchschnittsbürgerin oder der Durchschnittsbürger im Alltag wohl weniger mit Gesetzestexten als mit anderen Behördentexten konfrontiert sieht. 
[21] Trotzdem geht aus der Studie hervor, dass ein Grossteil der Bevölkerung die Rechtssprache als unverständlich einschätzt (ebd.: 6f.). Diese Einschätzung hängt jedoch nicht nur mit konkreten Erfahrungen im Umgang mit Rechtstexten zusammen, sondern ist auch gewissen Stereotypen über die juristische Sprache geschuldet (ebd.: 8). Zudem konnten sich nur wenige der befragten Personen spontan daran erinnern, dass die mangelnde Verständlichkeit der Texte konkrete Nachteile zur Folge gehabt hätte (ebd.: 11). Trotzdem spricht sich die Mehrheit der Befragten dafür aus, dass Allgemeinverständlichkeit Vorrang gegenüber der juristischen Fachsprache haben sollte (ebd.: 9).

[22] Diesen letzten Punkt kritisiert Szubert (2013: 54) mit dem Argument, dass sich die allgemeinsprachliche Bedeutung eines Wortes nicht unbedingt mit der Bedeutung in der juristischen Fachsprache deckt. So können beispielsweise gewisse Bedeutungskomponenten fehlen, oder man könnte dazu verleitet werden, ein Synonym zu verwenden, das zwar in der Alltagssprache dem ersetzten Wort entspricht, nicht jedoch in der Rechtssprache. Somit warnt Szubert vor zu einfachen, formelhaften Ansichten darüber, was verständlich sei.

[23] Die Kritik der Befragten an der Rechtssprache erschöpft sich jedoch nicht im Hinweis auf erklärungsbedürftige Fachtermini. Als Quellen für mangelnde Verständlichkeit wurden von den befragten Personen auch umständliche Formulierungen, Fremdwörter, eine abgehobene, unpersönliche oder veraltete Sprache sowie ein unhöflicher Ton genannt (ЕIснHoff-Cyrus et al. 2009: 10). Diese Kritikpunkte können redaktionell in Angriff genommen werden, ohne dass die fachsprachliche Exaktheit und somit die gesetzessprachliche Präzision darunter zu leiden hätte.

[24] Ebenfalls eher kritisch der Allgemeinsprachlichkeit gegenüber äussern sich MüLleR/ UhLmann (2013: 204). Sie argumentieren, dass sich das für Laiinnen und Laien relevante Recht nicht nur aus den Normtexten ergibt, sondern auch aus den Akten der Rechtsanwendung, welche die Normen konkretisieren. Durch die Rechtspraxis wird eine Norm mit Bedeutung angereichert (ebd.). Deshalb ist der Wortlaut von Erlasstexten nicht die alleinige Quelle der Normkenntnis, sondern bedarf der Auslegung (ОкsaAR 1989: 225). Ein allzu grosser Fokus auf die Allgemeinverständlichkeit von Erlasstexten könnte über diesen Umstand hinwegtäuschen und in den Laiinnen und Laien den Eindruck erwecken, dass der Wortlaut die Bedeutung einer Norm komplett abdeckt (vgl. Ogorek 2004: 299). Müller/Uhlmann (2013: 204f.) unterscheiden deshalb die aus den Gesetztestexten erlangte Rechtskenntnis von der strikten Normkenntnis und plädieren, wie auch die Richtlinien von Graubünden (GR:14), für die Priorität der Vermittelbarkeit gegenüber der Allgemeinverständlichkeit.

[25] Aus diesen Überlegungen geht auch hervor, dass Verständlichkeit in vielen Fällen nicht einfach eine Frage von «entweder oder» ist, sondern als etwas Skalares betrachtet werden muss (vgl. Lötscher 2016: 8f.; OKsaAr 1989: 231). Dieser Erkenntnis entspricht am ehesten die dritte der zu Beginn dieses Abschnitts genannten Positionen, die besagt, dass letztlich der Adressatenkreis massgeblich für die verwendete Sprache ist (vgl. ebd.: 229f.). Diese Position kann durch den folgenden Merksatz aus den Luzerner Richtlinien zusammengefasst werden: «Je breiter das Publikum ist, an das sich ein Erlass richtet, desto einfacher und näher beim üblichen Sprachgebrauch sollte die Erlasssprache sein» (LU:13). 


\section{Wortwahl}

[26] Die Wortwahl ist ein zentrales Thema der Gesetzestechnik, das von vielen kantonalen Richtlinien thematisiert wird. Im kommenden Unterabschnitt wird zunächst auf das Grundprinzip der lexikalischen Kohärenz eingegangen. Der zweite Unterabschnitt widmet sich der textsortenspezifischen Terminologie und der Kritik an alteingesessenen Formulierungen. Der letzte Unterabschnitt diskutiert schliesslich die direkte juristische Einflussnahme auf die Wortbedeutung mittels Legaldefinitionen.

\subsection{Lexikalische Kohärenz}

[27] Ein Grossteil der Richtlinien führt die lexikalische Kohärenz als ein Grundprinzip der Gesetzessprache an. ${ }^{18}$ Das Prinzip der lexikalischen Kohärenz besagt, dass dieselbe Sache stets mit demselben Ausdruck bezeichnet werden soll. Es kommt in den Richtlinien auf zwei Arten zum Ausdruck: Einerseits kann lexikalische Kohärenz vom Ausdruck her gedacht werden, was zum Grundsatz führt, dass ein Ausdruck stets im selben Sinne verwendet werden soll. ${ }^{19}$ Andererseits kann dasselbe Prinzip aber auch von der Sache her gedacht werden. In diesem Fall lautet der Grundsatz, dass für denselben Sachverhalt stets derselbe Ausdruck verwendet werden muss. ${ }^{20}$ Die folgenden exemplarischen Formulierungen aus den Richtlinien sollen diesen Unterschied veranschaulichen:

Vom Ausdruck her gedacht: «Es sind möglichst unzweideutige Begriffe zu wählen und diese in einem oder mehreren zusammenhängenden Erlassen stets mit dem gleichen Sinn zu verwenden.» (AG:7)

Von der Sache her gedacht: «Für die gleiche Sache sollen stets das gleiche Wort und die gleichen Bezeichnungen (Organe der Rechtspflege und der Verwaltung, Amtsstellen usw.) verwendet werden.» (TG:14)

[28] Auch wenn es sich hierbei um zwei Seiten desselben Prinzips handelt, ist es aus Sicht der Textproduktion doch sinnvoll, sich beider Aspekte bewusst zu sein. Diese stellen nämlich bis zu einem gewissen Grad voneinander unabhängige Fehlerquellen dar. Das übliche Verfahren beim Verfassen eines Textes besteht darin, einen bereits gefassten Gedanken sprachlich umzusetzen - man geht also von der Sache zum Begriff. Schematisch gesprochen bedeutet dies, dass man eine Sache A konsequent mit dem Ausdruck x und eine andere Sache B konsequent mit dem Ausdruck y bezeichnet. Ein Fehler könnte dann entstehen, wenn man übersieht, dass Ausdruck y auch noch für eine dritte Sache $C$ stehen könnte. Solche Fehler können vermieden werden, wenn man sich beider Aspekte der lexikalischen Kohärenz bewusst ist.

18 AG:7, GL:18, GR:14, UR:10, VS:7, BE:12, AR:7, AI:16, OW:11, LU:13, ZH:67, SG:48, TG:14.

19 AG:7, GL:18, GR:14, UR:10, VS:7, BE:12.

20 AR:7, AI:16, OW:11, LU:13, ZH:67, SG:48, TG:14. 


\subsection{Textsortenspezifische Terminologie}

[29] Wer einen Text verfasst, knüpft bewusst oder unbewusst an bestimmte sprachliche Muster an, die sich historisch für eine bestimmte Textsorte entwickelt haben. Solche Muster können, sofern sie den Leserinnen und Lesern vertraut sind, für das Textverständnis förderlich sein. Traditionelle Textmuster, wie etwa die Verwendung bestimmter Formulierungen, können dem Textverständnis aber auch abträglich sein, vor allem wenn sie unreflektiert verwendet werden. Es überrascht deshalb nicht, dass sich auch die kantonalen Richtlinien kritisch mit typisch gesetzessprachlichen Formulierungsmustern auseinandersetzen. Tabelle 1 trägt diese Kritikpunkte (und Lösungsvorschläge) zusammen:

\begin{tabular}{|c|c|c|}
\hline Funktion & Begriffe & Kommentar/Quelle \\
\hline \multirow[t]{2}{*}{ Derogation } & $\begin{array}{l}\text { in der Regel } \\
\text { regelmässig } \\
\text { grundsätzlich } \\
\text { ausnahmsweise }\end{array}$ & $\begin{array}{l}\text { Sollen vermieden werden (BE:18, GL:26). } \\
\text { Vorschlag: Umstände konkretisieren (ZH:67, } \\
\text { BE:18) und andere Regelungstechniken wie nicht } \\
\text { abschliessende Aufzählungen oder } \\
\text { Generalklauseln verwenden (GL:26, } \\
\text { vgl. Bundesamt für Justiz 2009: 6f.). }\end{array}$ \\
\hline & Vorbehalt & $\begin{array}{l}\text { Bedingt: Nur zu verwenden, um Unklarheiten } \\
\text { zu beseitigen oder wenn sich die Ausnahme } \\
\text { nicht bereits aus dem Text ergibt. Kann durch } \\
\text { die logische Struktur des Erlasses vermieden } \\
\text { werden (BE:16). } \\
\text { Anmerkung: Die Relevanz von Vorbehalten } \\
\text { sollte aber nicht vernachlässigt werden. Sie } \\
\text { besagen, dass eine andere als die vorliegende } \\
\text { Norm im Kollisionsfall vorgeht. }\end{array}$ \\
\hline \multirow[t]{2}{*}{ Verknüpfungen } & $\begin{array}{l}\text { ferner } \\
\text { daher } \\
\text { ebenso }\end{array}$ & $\begin{array}{l}\text { Sollen vermieden werden (VS:7, BE:3). } \\
\text { (Ferner und ebenso stellen neutrale } \\
\text { Verknüpfungen dar, die in einem ohnehin stark } \\
\text { strukturierten Erlasstext weggelassen werden } \\
\text { können. Daher, welches einen Grund mit einer } \\
\text { Folge verknüpft, macht in einem Normtext } \\
\text { wenig Sinn, in dem ja nur geregelt und nicht } \\
\text { begründet werden soll.) }\end{array}$ \\
\hline & und/oder & $\begin{array}{l}\text { Soll vermieden werden. } \\
\text { Vorschlag: Grundsätzlich durch oder zu ersetzen. } \\
\text { Falls dies zu einer ungewollten Bedeutung führt, } \\
\text { beide Fälle getrennt darstellen (AI:17, BE:16). }\end{array}$ \\
\hline
\end{tabular}




\begin{tabular}{|c|c|c|}
\hline Funktion & Begriffe & Kommentar/Quelle \\
\hline Verknüpfungen & beziehungsweise & $\begin{array}{l}\text { Bedingt: Nur zu verwenden, wenn vorhergehend } \\
\text { eine Unterscheidung eingeführt worden ist und } \\
\text { die Alternativen nun mit unterschiedlichen } \\
\text { Ergänzungen versehen werden (ZH:70). } \\
\text { (Schematisch: «Für A und B gilt x respektive y» } \\
\text { bedeutet, dass x für A gilt und y für B. Dagegen } \\
\text { wäre bei «Für A und B gilt x oder y» möglich, } \\
\text { dass y auch für A gilt.) } \\
\text { Wenn keine solche Unterscheidung getroffen } \\
\text { wird, kann beziehungsweise meist durch oder } \\
\text { ersetzt werden (BE:16). } \\
\text { (Schematisch: «Für A gilt x beziehungsweise y» } \\
\text { ist gleichbedeutend mit «Für A gilt x oder y»). }\end{array}$ \\
\hline $\begin{array}{l}\text { Sinngemässe } \\
\text { Anwendung }\end{array}$ & $\begin{array}{l}\text { sinngemäss } \\
\text { analog }\end{array}$ & $\begin{array}{l}\text { Mit Vorsicht zu verwenden (ZH:69f.), da relativ } \\
\text { schnell Unklarheiten entstehen können. } \\
\text { Darf nicht verwendet werden, wenn die Norm } \\
\text { unverändert anwendbar ist (BE:16). }\end{array}$ \\
\hline \multirow[t]{2}{*}{ Bedingungen } & Wenn, sofern & $\begin{array}{l}\text { Absolute Bedingung (entweder erfüllt oder } \\
\text { nicht) (ZH:69, SG:47). }\end{array}$ \\
\hline & soweit & $\begin{array}{l}\text { Relative Bedingung (in dem Masse, wie die } \\
\text { Voraussetzungen erfüllt sind) (ZH:69, SG:47). }\end{array}$ \\
\hline
\end{tabular}

Tabelle 1: Übersicht zu den Hinweisen der kantonalen Richtlinien über textsortenspezifische Ausdrücke.

[30] In der sprachwissenschaftlichen Literatur findet sich der Grundsatz, dass von der Allgemeinsprache nur dann abgewichen werden soll, wenn es unbedingt nötig ist (Gesellschaft für deutsche Sprache 1998: 36). Typisch für die Gesetzessprache ist laut der Gesellschaft für deutsche Sprache auch die Tendenz, Ausdrücke länger zu machen, als nötig wäre. Dies zeige sich etwa in der Verwendung unnötiger Vorsilben (abkaufen und einsparen anstelle von kaufen und sparen, ebd.: 50f.) oder redundanter Wortbestandteile (im Bedarfsfall anstelle von bei Bedarf oder Schreinereibetrieb anstelle von Schreinerei, ebd.: 41). Ein weiteres Phänomen in diesem Zusammenhang seien die sogenannten Streckverben (auch Funktionsverbgefüge genannt), bei denen eine Wortbedeutung quasi auf zwei Wörter aufgeteilt wird, beispielsweise in Anrechnung bringen anstelle von anrechnen oder zur Annahme gelangen anstelle von angenommen werden (ebd.: 44).

[31] Solche Empfehlungen sind aber stets mit Vorsicht zu geniessen, denn bei Vereinfachungen besteht auch die Gefahr, dass relevante Unterscheidungen wegfallen. So ist es etwa nicht ganz abwegig, zwischen Schreinereibetrieb und Schreinerei zu unterscheiden, wenn mit ersterem die Firma, also die juristische Person, mit letzterem aber die Werkstatt im Sinne einer Lokalität gemeint ist. Und auch Streckverben können sinnvoll sein, etwa dann, wenn man in der Formulierung das Akkusativobjekt weglassen will. So ist ein Satz wie Das Amt genehmigt ungrammatisch, weil stets etwas genehmigt werden muss. Grammatisch korrekt ist dagegen Das Amt erteilt die Genehmi- 
gung (ebd. 1998: 45). In welchem Kontext eine solche Formulierung sinnvoll ist, ist eine Frage der Textstruktur.

\subsection{Legaldefinitionen}

[32] Die Gesetzessprache kann die Bedeutung von Begriffen durch so genannte Legaldefinitionen präzisieren. Allerdings weisen die Richtlinien darauf hin, dass dieses Mittel nur sparsam eingesetzt werden soll (z. B. ZG:15, ZH:18). Dieser Grundsatz ist durchaus sinnvoll, denn das Bedürfnis nach exakten Rechtsbegriffen (das ja auch in der Bevölkerung spürbar ist) könnte zu einer Art Dammbruch führen: Da Sprache naturgemäss vage und mehrdeutig ist, kommt man schnell zum Schluss, dass im Grunde jeder Begriff definitionswürdig wäre. Zudem droht die Gefahr, dass man in einen infiniten Prozess des Definierens gerät, da die zur Definition herangezogenen Begriffe ja ihrerseits wieder definiert werden müssten. Sinnvoller ist es da, von Anfang an auf die Sprache zu vertrauen (vgl. Nussbaumer 2008: 318-321) und Legaldefinitionen von spezifischen Funktionen abhängig zu machen:

- zur Klärung mehrdeutiger, unklarer oder nicht allgemein verständlicher Begriffe, insbesondere auch fachspezifischer Begriffe (ZH:18/75, GL:18/20, SO:10, ZG:15, SG:25/49, LU:5, GR:17, TG:13).

- wenn eine Vereinheitlichung der Begriffe zu einer Erleichterung bei der Rechtsanwendung führt (ZH:18, GL:20). Ein Beispiel dafür wird in den Richtlinien nicht angeführt. Wahrscheinlich ist damit gemeint, dass durch Legaldefinitionen auch die begriffliche Kohärenz (vgl. Abschnitt 3.1) gefördert werden kann.

- zur Verkürzung des Erlasses (ZH:18, GL:18/20, ZG:34, SG:25). Damit ist gemeint, dass mittels Legaldefinitionen lange Ausdrücke durch kürzere Ausdrücke ersetzt werden können, was der Textökonomie zugutekommt.

[33] Des Weiteren werden in den Richtlinien Prinzipien angeführt, welche die Entscheidung erleichtern sollen, ob nun im einzelnen Falle eine Legaldefinition verwendet werden soll oder nicht:

- Frequenz des Begriffs: Je häufiger ein Begriff ist, desto eher soll er definiert werden. So führen die Richtlinien von Zug und St. Gallen eine dreimalige Verwendung als Mindestfrequenz an (ZG:15, SG:25).

- Relevanz des Begriffs: Je zentraler der Begriff ist, desto eher soll er definiert werden (ZG:15).

[34] Wichtig ist, dass man diese Prinzipien nicht als alleinige Entscheidungsgrundlage auffasst, sondern eben nur als ein mögliches Kriterium. In die Entscheidung sollen auch ganz pragmatische Überlegungen einfliessen: Ein Begriff muss nicht zwangsläufig definiert werden, nur weil er in einem Erlass häufig vorkommt. Es kommt auch darauf an, wie «aufwendig» die eigentliche Beschreibung ist, die man durch eine Legaldefinition ersetzen würde (vgl. BRATsCHI 2009: 194f.). Wenn sie eher kurz ist, erübrigt sich eine Definition. Ferner muss ein alltäglicher und verständlicher Begriff nicht automatisch definiert werden, nur weil er im Zentrum eines Erlasses steht und häufig vorkommt. 
[35] Neben ihrer Funktion werden in den kantonalen Richtlinien noch drei weitere Aspekte der Legaldefinition geregelt: ihre Position im Text, die Reihenfolge bei mehreren Definitionen und schliesslich ihre Form.

[36] Was die Position betrifft, so sei eine Legaldefinition grundsätzlich im Einleitungsteil oder - wenn der Begriff nur für eine bestimmte Gliederungseinheit verwendet wird - zu Beginn der betroffenen Gliederungseinheit zu positionieren (z. B. ZH:17/19, SO:10). Letzteres ist vor allem bei längeren Erlassen sinnvoll. Problematisch wäre es, diese beiden Ansätze zu mischen: Ein Begriffsartikel zu Beginn des Textes lässt annehmen, dass dieser alle Legaldefinitionen des Erlasses enthält. Allfällige spätere, abschnittsspezifische Legaldefinitionen wirken irritierend oder können übersehen werden.

[37] Für den Fall, dass es durch diese Positionierung dazu kommt, dass mehrere Legaldefinitionen aufeinanderfolgen, werden verschiedene Organisationsprinzipien vorgeschlagen:

- Inhaltliche Logik: Oberbegriffe gehen den Unterbegriffen voraus (SG:26, GL:20)

- die Reihenfolge entspricht der Abfolge der Begriffe im Erlass (SG:26, GL:20)

- alphabetische Reihenfolge (SG:26)

[38] Welchem dieser drei Systeme soll nun der Vorzug gegeben werden? Die Glarner Richtlinien ziehen die begriffshierarchische Gliederung derjenigen der Begriffsabfolge vor (GL:20). Als am leserfreundlichsten dürfte sich allerdings das Prinzip der alphabetischen Reihenfolge erweisen - dies umso mehr, je länger die Liste wird. Die Leserinnen und Leser suchen in den meisten Fällen nach einem bestimmten Begriff und können ihn so unkompliziert auffinden. Falls eine Hierarchisierung der Begriffe für das Textverständnis unumgänglich sein sollte, böte sich eine alphabetische Gliederung auf Ebene der Oberbegriffe an, wobei die Unterbegriffe jedem Oberbegriff in der nächstunteren Gliederungsebene angehängt und ihrerseits wieder alphabetisch geordnet werden können. Das alphabetische Gliederungsprinzip muss aber dahingehend relativiert werden, dass es nur bei Erlassen sinnvoll ist, die einsprachig vorliegen. Bei mehrsprachigen Erlassen führt es zu unterschiedlichen Reihenfolgen und kann somit nicht angewendet werden.

[39] Ein weiteres Ordnungsprinzip, das in den kantonalen Richtlinien nicht direkt erwähnt wird, ist die thematische Ordnung. Sie ist mit der inhaltlichen Logik verwandt, bezieht sich aber weniger auf das Verhältnis von Ober- und Unterbegriffen, sondern auf die thematische Zusammengehörigkeit der Begriffe.

[40] Was die Form betrifft, so muss die explizite Definition von der Klammerdefinition unterschieden werden (ZH:19). Der Unterschied liegt darin, dass die Klammerdefinition im Gegensatz zur expliziten Definition keinen eigenen Satz darstellt, sondern gewissermassen in einen anderen Satz integriert ist (vgl. BRATSCH 2009: 194). Sie ist somit weniger einfach auffindbar und geht von einer linearen Lektüre aus. Klammerdefinitionen eignen sich deshalb besser für kürzere Erlasse oder Definitionen, die nur für einen klar begrenzbaren Textabschnitt (z. B. einen Artikel) relevant sind, wo von einer linearen Lektüre ausgegangen werden kann.

[41] Bezüglich des Aufbaus sprechen die Zuger Richtlinien den zweiteiligen Aufbau einer Definition an und verweisen dabei auf das Grundprinzip, dass der zu definierende Begriff (Definiendum) dem beschreibenden Element (Definiens) vorausgehen soll (ZG:15). Beim Definiens handle es sich dann entweder um eine Umschreibung der Bedeutung des Begriffs oder um eine Aufzählung von Dingen, die er umfasst. Dabei sollen Redundanzen, Tautologien und Pleonas- 
men vermieden werden (ZG:15). Wichtig ist zudem, dass das Definiendum nicht mit sich selbst erklärt wird - das Definiendum darf also nicht im Definiens enthalten sein (ZG:15).

[42] Die Literatur nennt weitere Gütekriterien, die Legaldefinitionen erfüllen müssen. So führt Bratschi (2009) die folgenden Faustregeln an:

- «Ein definierter Begriff ist innerhalb eines Erlasses immer im definierten Sinn zu verwenden» (ebd.: 202).

- «Einem definierten Begriff darf nur eine Bedeutung zugewiesen werden» (ebd.: 204).

- «Die Definition soll (in einem vernünftigen Mass) präzise sein» (ebd.: 207).

- «Die Definition soll auf lautere Begriffe zurückgreifen» (ebd.: 208).

- «Die Definition soll keine materiellen Elemente enthalten» (ebd.: 209).

[43] Zudem sollte darauf geachtet werden, dass im Grundsatz an jeder Textstelle, an der das Definiendum vorkommt, auch das Definiens stehen könnte, ohne dass es zu sprachlichen Fehlern oder inhaltlichen Widersprüchen kommt.

\section{Satzbau: Informationsdichte}

[44] Wie aus Abschnitt 2 hervorgegangen ist, sollte die Gesetzessprache möglichst einfach und verständlich sein, ohne aber den Regelungsgegenstand an sich zu vereinfachen. Auf der Ebene des Satzes bedeutet dies zweierlei: Einerseits müssen komplexe Sachverhalte so segmentiert werden, dass in jedem Satz idealerweise genau ein Aspekt der Gesamtregelung zum Ausdruck kommt. Andererseits muss die sprachliche Form diesen Aspekt möglichst einfach und verständlich darstellen. In diesem Abschnitt wird auf den ersten Aspekt eingegangen, indem die Frage nach der optimalen Informationsdichte eines Rechtssatzes gestellt wird. Der zweite Aspekt, die sprachliche Komplexität, wird in Abschnitt 5 behandelt.

[45] Der dritte Teil der Eugen-Huber-Regel (vgl. Abschnitt 2.1) besagt, dass pro Satz ein Gedanke ausgedrückt werden soll. Dieser Anspruch kommt nicht nur der Verständlichkeit entgegen, sondern ist auch aus juristischer Sicht sinnvoll, da so verhindert werden kann, dass in einem Satz mehrere Normen gleichzeitig zum Ausdruck kommen (vgl. Höfler 2011). Eine der zentralen Herausforderungen beim Verfassen von Erlassen besteht also darin, einen komplexen Sachverhalt in einzelne «Gedanken» zu segmentieren. Dabei kann es hilfreich sein, den Sachverhalt auf eine Reihe von Regelungseinheiten herunterzubrechen, die dann jeweils in einem Satz ausgedrückt werden. Diese Regelungseinheiten können verschiedenen Kategorien zugeordnet werden. Die Zürcher Richtlinien schlagen folgende Kategorien vor (ZH:72):

1. wenn-dann-Konstruktion (Tatbestand und Rechtsfolge)

2. Verfahrensfragen

3. Zusammensetzung eines Organs

4. Wahl eines Organs

5. Aufgaben (Zuständigkeiten) eines Organs

6. Aufsicht 
[46] Beim Verschriftlichen der Inhalte ist es aber schnell passiert, dass ein Satz mehr als nur einen Gedanken enthält. Dem dritten Teil der Eugen-Huber-Regel steht bis zu einem gewissen Grad der Anspruch der Knappheit entgegen, denn wer kurze und scheinbar einfache Sätze schreiben will, läuft schnell Gefahr, zu viel Information in einem einzelnen Satz zu komprimieren. Da die Sprache eine Vielzahl an Komprimierungsstrategien zur Verfügung stellt, geschieht dies häufig unbewusst. Es gilt also, sich der möglichen Gefahren des kompakten Schreibens bewusst zu sein. Im Folgenden werden mit Ellipsen, dem Nominalstil und multipropositionalen Sätzen drei solcher Gefahrenquellen besprochen.

\subsection{Ellipsen}

[47] Wer sich in einer Materie auskennt, denkt vieles mit, ohne es explizit auszudrücken. Die Sprache erlaubt es häufig, Mitgedachtes einfach wegzulassen. Man spricht dabei von sogenannten Ellipsen. Was in der Alltagskommunikation ein Vorteil sein kann, wird in der Gesetzessprache zum Nachteil: Durch allzu sparsames Formulieren können Bezüge unklar oder gar unlogisch werden. In solchen Fällen kann es sinnvoll sein, etwas längere und dafür exaktere Formulierungen in Kauf nehmen (vgl. Hartmann/Parádi 2018: 219-222). Dies soll an einem Beispiel (ebd.: 219) aufgezeigt werden:

Soweit es zur Sicherstellung der Grundversorgung in einer Versorgungskrise geboten ist, können durch Rechtverordnung Vorschriften erlassen werden über [...]

4. die Verwendung von Maschinen und Geräten, Treibstoffen, Brennstoffen, Geräten zur Notstromversorgung sowie von sonstigen Betriebsmitteln zum Herstellen oder Behandeln von Erzeugnissen.

[48] Dieses Beispiel enthält zwei potenzielle Ellipsen. Die Erste ist offenkundig und entspricht dem Ausdruck die Verwendung von. Dieser bezieht sich zunächst auf Maschinen und Geräte, kann aber geistig auch bei den nachfolgenden Gliedern der Aufzählung hinzugefügt werden. Beim zweiten Fall, dem Ausdruck zum Herstellen oder Behandeln von Erzeugnissen, ist jedoch nicht klar, ob es sich um eine Ellipse handelt. Aus dem Wortlaut des Satzes geht nicht hervor, ob sich dieser Ausdruck nur auf das letzte Glied der Aufzählung (von sonstigen Betriebsmitteln) oder auf alle vorangehenden Glieder bezieht. Um diese Frage zu klären, müssen die Erläuterungen zur Norm herangezogen werden. Eine Rechtsnorm sollte allerdings immer ohne Konsultation der Erläuterungen verständlich sein.

[49] Unter Berücksichtigung der Erläuterungen, aus denen hervorgeht, dass sich das Attribut auf alle vorangehenden Aufzählungsglieder bezieht, schlagen Hartmann/Parádi (2018: 221) folgende Lösung vor:

\section{4. die Verwendung von}

a. Maschinen und Geräten zum Herstellen oder Behandeln von Erzeugnissen,

b. Treibstoffen und Brennstoffen für diese Maschinen und Geräte,

c. Geräten zur Notstromversorgung zum Betrieb dieser Maschinen und Geräte sowie

d. sonstigen Betriebsmitteln zum Herstellen oder Behandeln von Erzeugnissen. 
[50] In diesem Lösungsvorschlag wird das Attribut wiederholt (Buchstaben a und d) oder ausformuliert (Buchstaben b und c).

\subsection{Nominalstil}

[51] Für die Gesetzessprache typisch, jedoch nicht immer gewinnbringend, ist der sogenannte Nominalstil (vgl. Nussbaumer 2009: 2138). Er zeichnet sich durch eine Häufung von Nomen und dadurch aus, dass Informationseinheiten, die üblicherweise als Nebensätze realisiert werden, in Form von Nominalphrasen in den übergeordneten Satz integriert werden (Bussmann 2008: 480). Deshalb ermöglicht der Nominalstil kompakte Formulierungen, wie das folgende Beispiel zeigt (Hartmann/Parádi 2018: 227):

Die Genehmigung ist ferner zu erteilen, wenn [... ] die Änderung einer baulichen Anlage der Energieeinsparung durch Anpassung an die baulichen oder anlagentechnischen Mindestanforderungen der Energieeinsparverordnung dient [...]

[52] Eine solche Formulierung ist jedoch problematisch (ebd.: 225-229). Aufgrund der beschränkten Kasusmarkierung des Deutschen sind Bezüge zwischen den einzelnen Phrasen nicht klar. Sowohl einer baulichen Anlage, der Energieeinsparungen wie auch der Energieeinsparverordnung könnten - rein formal gesehen - im Genitiv oder aber im Dativ stehen. Je nach dem handelt es sich um ein Genitivattribut der vorangehenden Phrase oder um das Dativobjekt des Verbs dienen. Dass dieses durch die lange Kette von Nominalphrasen erst ganz am Schluss des Satzes steht (sogenannte Satzklammer), erschwert die Interpretation zusätzlich, denn es ist das Verb, das die Struktur des Satzes vorgibt.

[53] Diese Problematik kann entschärft werden, indem ein Nebensatz eingeführt wird, der die Satzklammer auflöst und die Bezüge zwischen den einzelnen Phrasen verdeutlicht (ebd.: 228):

Die Genehmigung ist ferner zu erteilen, wenn [... ] die Änderung einer baulichen Anlage dazu dient, durch Anpassung an die baulichen oder anlagentechnischen Mindestanforderungen der Energieeinsparverordnung Energie einzusparen.

[54] Der Nominalstil hat aber auch Vorteile (vgl. Gesellschaft für deutsche Sprache 1998: 37), etwa wenn er dem Weglassen des Agens dient (Deagentivierung) oder wenn das Verb gegenüber der Nominalkonstruktion zu vage oder mehrdeutig ist (z. B. bei widersprechen gegenüber Widerspruch einlegen.)

\subsection{Multipropositionale Sätze}

[55] Multipropositionale Sätze sind Sätze, die mehrere Regelungsaussagen beinhalten. Sie wirken auf den ersten Blick übersichtlich und verständlich, können jedoch den Leserinnen und Lesern die Lektüre auch erschweren, da die einzelnen Aussagen herausgearbeitet werden müssen. Schlimmstenfalls kommt es zu falschen oder unlogischen Bezügen oder sogar zu einer ungenügenden Grundlage für staatliches Handeln (vgl. Höfler 2017: 166-169). Hier lohnt es sich, längere und allenfalls auch repetitive Strukturen in Kauf zu nehmen (vgl. Hartmann/Parádi 2018: 222-225). 
[56] HöFLER (2011) diskutiert verschiedene Kategorien von multipropositionalen Sätzen und führt Strategien an, mit denen diese aufgelöst werden können. In diesem Abschnitt wird auf die letzten beiden dieser Kategorien eingegangen, da diese am schwierigsten aufzulösen sind: die Adverbialien und die Attribute. Die Schwierigkeit liegt darin, dass nicht jedes Adverbiale und jedes Attribut zwangsläufig zu einem multipropositionalen Satz führt.

[57] Adverbialien sind Satzglieder, die den ganzen Satz modifizieren (vgl. Höfler 2011: 269-273) und nicht mit der Wortart der Adverbien zu verwechseln sind. Sie umfassen häufig mehrere Wörter und treten beispielsweise als Präpositionalphrase in Erscheinung, wie folgendes Beispiel (ebd.: 269) zeigt:

Der Bundesrat kann auf der Grundlage der voraussichtlichen Aufwendungen eine Pauschale festlegen.

[58] Nicht alle Adverbialien führen zu multipropositionalen Sätzen. Es muss zwischen adverbialen Angaben und adverbialen Ergänzungen unterschieden werden. Während adverbiale Angaben eine zusätzliche Information ausdrücken, sind adverbiale Ergänzungen ein notwendiger Bestandteil der Aussage. Ob etwas ein notwendiger Bestandteil der Aussage ist, hängt massgeblich vom Verb ab und kann mit der Weglassprobe ermittelt werden. Während das Adverbiale im obigen Beispiel eine Angabe ist, ist dasjenige im folgenden Beispiel (ebd.) eine Ergänzung:

Die Beiträge [...] werden auf der Grundlage der anrechenbaren Gesamtprojektkosten bemessen.

[59] Nur adverbiale Angaben, nicht jedoch adverbiale Ergänzungen führen zu multipropositionalen Sätzen und sollten deshalb in eigene Sätze ausgegliedert werden, wie folgendes Beispiel (ebd.) zeigt:

Der Bundesrat kann eine Pauschale festlegen. Er stützt sich dabei auf die voraussichtlichen Aufwendungen.

[60] Rein grammatikalisch gesehen, können auch adverbiale Ergänzungen in eigene Sätze ausgelagert werden. Dies ist im Normalfall jedoch nicht sinnvoll, da so lediglich der Inhalt eines Satzes auf zwei Sätze verteilt wird, wie folgendes Beispiel (ebd.) zeigt:

Die Beiträge [...] werden bemessen. Die Bemessung erfolgt auf der Grundlage der anrechenbaren Gesamtprojektkosten.

[61] Ein Beispiel aus der Alltagssprache soll dieses Prinzip noch verdeutlichen:

Max isst etwas. Es ist ein Apfel.

[62] Die zweite Kategorie, die hier behandelt werden soll, sind die Attribute (vgl. ebd.: 274f.). Attribute sind Elemente, die ein Nomen näher beschreiben. Wie schon bei den Adverbialien, führt nicht jedes Attribut zu einem multipropositionalen Satz. Auch hier stellt sich die Frage, ob es eine integrale, definierende Bedeutungskomponente ausdrückt (sogenannte restriktive Verwendung) oder ob es lediglich eine Zusatzinformation darstellt (nicht-restriktive Verwendung). Zusatzinformationen können in eigenständige Sätze ausgegliedert werden, restriktive Attribute nicht. Im folgenden Beispiel bringt das Attribut (der unterstrichene Relativsatz) eine Zusatzinformation zum Ausdruck. Es ist demgemäss ein nicht-restriktives Attribut und kann in einen eigenen Satz ausgegliedert werden: 
Attribut: Auf Antrag des Fakultätsvorstands setzt die Universitätsleitung eine Berufungskommission ein, der mindestens zwei externe Expertinnen oder Experten angehören.

Eigener Satz: Auf Antrag des Fakultätsvorstands setzt die Universitätsleitung eine Berufungskommission ein; der Berufungskommission gehören mindestens zwei externe Expertinnen oder Experten an.

[63] Das Attribut wird in einen eigenen Satz ausgelagert, indem der Relativsatz aufgelöst wird. Dazu wird das Bezugswort (Berufungskommission) im neuen Satz wiederholt. Diese Strategie lässt sich auch auf eine Vielzahl anderer Attributtypen anwenden.

\section{Satzbau: Sprachliche Komplexität}

[64] Auch Sätze, die nur einen Gedanken beinhalten, können recht komplex sein. Der Anspruch der Einfachheit und Verständlichkeit erschöpft sich deshalb nicht im Segmentieren der Information, sondern betrifft auch die sprachliche Struktur, mit der die Information ausgedrückt wird. Es stellt sich also die Frage, wie man den Aufbau eines Satzes vereinfachen kann, ohne dass sein Informationsgehalt beeinträchtigt wird. Eine solche Vereinfachung stellt das folgende Beispiel aus den Berner Richtlinien dar (BE:8):

Ursprünglich: Jedermann hat Anspruch auf Geheimhaltung der ihn betreffenden personenbezogenen Daten.

Verändert: Jedermann hat das Recht auf Geheimhaltung der personenbezogenen Daten, die ihn betreffen.

[65] Indem die Partizipialkonstruktion (ihn betreffenden) in einen Relativsatz (die ihn betreffen) umgewandelt wird, wächst die Formulierung um ein Wort. Dennoch ist der zweite Satz verständlicher, weil das beschreibende Element auf das beschriebene Element (die personenbezogenen Daten) folgt: Da der Träger der Eigenschaft bereits bekannt ist, kann die Eigenschaft sogleich zugeordnet werden. Diese Umstrukturierung wird umso dringender, je länger der Satz ist.

[66] Auf kognitiver Ebene bedeuten solche Umformulierungen eine Vereinfachung, weil die Eigenschaft nicht als separate Informationseinheit «mitgedacht» werden muss, sondern mit ihrem Träger zu einer Informationseinheit zusammengefasst wird (sogenannte Unifikation, vgl. HaGoort/Indefrey 2014). Kognitiv verarbeitet werden nämlich, worauf empirische Untersuchungen hinweisen, nicht nur einzelne Wörter, sondern auch Bedeutungseinheiten, die mehrere Wörter umfassen können (vgl. NeLson et al. 2017).

[67] In diesem Abschnitt wird zunächst besprochen, welche Änderungen der sprachlichen Komplexität eines Rechtssatzes überhaupt sinnvoll sind. Danach wird mit dem Passiv eine viel kritisierte Satzstruktur diskutiert, bevor schliesslich auf die Perspektive eingegangen wird, aus der die Sätze formuliert werden können.

\subsection{Sinnvolle und weniger sinnvolle Änderungen}

[68] Nicht alle Änderungen im Hinblick auf die sprachliche Komplexität eines Rechtssatzes sind gleichermassen sinnvoll. Wie das obige Beispiel gezeigt hat, zeichnen sich sinnvolle Änderungen 
nicht bloss durch die Reduktion der Anzahl Wörter einer Formulierung aus, sondern dadurch, dass den Leserinnen und Lesern das Verarbeiten der Information erleichtert wird.

[69] Jedoch stellt nicht jede Verkürzung automatisch eine Vereinfachung dar. Ein Beispiel dafür ist die folgende Umformulierung, die in den Berner Richtlinien angeführt wird (BE:8):

Ursprünglich: Gemeinden, die mehr als 10000 Einwohner haben

Verändert: Gemeinden mit mehr als 10000 Einwohnern

[70] Durch die Umformulierung des Relativsatzes als Präpositionalkonstruktion kann zwar das Verb haben weggelassen werden, doch die Struktur des Ausdrucks bleibt dieselbe: Auf das allgemeine Merkmal (Gemeinden) folgt eine Spezifizierung (mit mehr als 10000 Einwohnern).

[71] Solche Verkürzungen verbessern zwar nicht die Verständlichkeit einer Formulierung, sie fügen ihr jedoch auch keinen Schaden zu. Vorsicht geboten ist dagegen beim Weglassen sogenannter überflüssiger Elemente, wie es die Gesellschaft für Deutsche Sprache in ihren «Fingerzeigen für die Gesetzes- und Amtssprache» empfiehlt. Gemeint sind damit wohl Ausdrücke, die nicht notwendig sind, um die jeweilige Sache hinreichend zu definieren. Die Gefahr beim Weglassen überflüssiger Elemente besteht darin, dass die weggelassenen Elemente nur scheinbar überflüssig sind. Das heisst aber nicht, dass in vielen Fällen tatsächlich Vereinfachungen vorgenommen werden können, wie die folgenden Beispiele zeigen:

1. die getroffenen Vereinbarungen > die Vereinbarungen (Gesellschaft für deutsche Sprache 1998: 33)

2. in der Absicht, etwas stehlen zu wollen > In der Absicht, etwas zu stehlen (ebd.: 33)

3. Schreinereibetrieb $>$ Schreinerei (ebd.: 41).

4. zeitlich befristeter Vertrag > befristeter Vertrag (ebd.: 33)

\subsection{Die Causa Passivsatz}

[72] Der Passivsatz nimmt in vielen Richtlinien eine Sonderstellung ein. Der Tenor ist eindeutig: Der Aktivsatz sei grundsätzlich vorzuziehen oder das Passiv sei zumindest mit Mass zu verwenden. ${ }^{21}$ Die Richtlinien von St. Gallen weisen darauf hin, dass bei der Verwendung des Passivs darauf zu achten sei, dass der Normadressat stets klar aus dem Kontext hervorgehe (SG:47). Genau dieser Umstand, dass nämlich beim Passivsatz etwas Implizites hergeleitet werden muss und allenfalls sogar der Normadressat unklar ist, mag der Ursprung für das weit verbreitete Ressentiment gegen diese grammatikalische Konstruktion sein.

[73] Beim Passiv handelt es sich, linguistisch betrachtet, um eine Veränderung in der sogenannten Verbvalenz. Aus einem zweiwertigen Verb $(A$ macht $B)$ wird ein einwertiges: $B$ wird gemacht. Das Agens A (d. h. die handelnde Instanz) ist zwar auf der Ebene der Bedeutung noch da und kann auch mittels Präpositionalergänzung ausgedrückt werden ( $B$ wird von $A$ gemacht), doch es rückt gegenüber dem Objekt $B$ in den Hintergrund. Diese Struktur ist an sich weder gut noch schlecht, es stellt sich lediglich die Frage, wann ihr Einsatz sinnvoll ist.

21 ZH:74, UR:10, OW:10, GL:18, ZG:35, VS:7. 
[74] Darauf gehen die Berner Richtlinien näher ein und nennen drei Fälle, in denen der Einsatz des Passivs sinnvoll sei (BE:10). Der erste dieser Fälle ist die Verpflichtung einer Vielzahl nicht näher bestimmter Personen. Hier könnte man zudem anfügen, dass in diesem Kontext der Passivsatz sogar eleganter und verständlicher ist als sein Aktiväquivalent, welches auf bedeutungsarme Begriffe wie alle, jede Person oder jedermann zurückgreifen müsste. Solche Ausdrücke erschweren nur den Blick auf das Wesentliche, nämlich die Handlung selbst. Eine Ausnahme bilden Verfassungsgrundsätze, deren Allgemeingültigkeit aus Gründen der Symbolik hervorgehoben werden soll. Ein Beispiel dafür ist folgender Satz aus der Verfassung des Kantons Zürich:

Jede Person nimmt Verantwortung für sich selber wahr und trägt nach ihren Kräften zur Bewältigung der Aufgaben in Staat und Gesellschaft bei. (Art. 5 Abs. 1 Verfassung des Kantons Zürich ${ }^{22}$ )

[75] Der zweite Fall trifft zu, wenn das Agens (die handelnde Instanz) nicht weiter spezifiziert werden kann oder seine Spezifikation unwichtig ist. Das folgende Beispiel (BE:10) soll dies veranschaulichen:

Für Dienstleistungen, die auf Ersuchen hin dringlich oder ausserhalb der normalen Arbeitszeit verrichtet werden, können Zuschläge bis zu 50 Prozent erhoben werden.

[76] Dieses Beispiel wirft die Frage auf, was genau mit Spezifikation gemeint ist. Denn damit die Norm sinnvoll ist, müsste durchaus klar sein, wer Zuschläge bis zu 50 Prozent erheben kann. Um wen es sich dabei handelt, muss jedoch aus den umliegenden Sätzen abgeleitet werden. Der Passivsatz erfüllt hier also eine ökonomische Funktion: Da das Agens aus dem Kontext bereits bekannt ist, muss es nicht nochmals wiederholt werden.

[77] Der dritte der in den Berner Richtlinien genannten Fälle ist das Hervorheben des Themas und das damit verbundene Anknüpfen an vorangehende Sätze. Das folgende Beispiel (BE:10) soll dies veranschaulichen:

Der Regierungsrat setzt den Teuerungsausgleich auf Grund der jeweiligen Lebenskosten auf den 1. Januar im Verhältnis zu den massgebenden Bezügen fest.

Der Teuerungsausgleich wird vom Regierungsrat auf Grund der jeweiligen Lebenskosten auf den 1. Januar im Verhältnis zu den massgebenden Bezügen festgesetzt.

[78] Durch den Passivsatz wird der Gegenstand der Handlung (Teuerungsausgleich) zum Thema gemacht, während das Agens (Regierungsrat) in den Hintergrund tritt. Obwohl nach wie vor eine Handlung des Regierungsrats geschildert wird, trifft der Rechtssatz jetzt in erster Linie eine Aussage über den Teuerungsausgleich. Der Sinn einer solchen Konstruktion liegt vor allem in der Verknüpfung der Rechtssätze untereinander. Aus den Berner Richtlinien geht nicht hervor, welcher Rechtssatz diesem Beispiel unmittelbar hervorgeht. Das Thematisieren des Teuerungsausgleichs ist jedoch vor allem dann sinnvoll, wenn im vorangehenden Satz bereits vom Teuerungsausgleich die Rede war und nun daran angeschlossen werden kann (sogenannte Thema-Rhema-Struktur, vgl. HÖFLER 2016: 233-236).

22 Verfassung vom 27. Februar 2005 des Kantons Zürich (SR 131.211). 


\subsection{Perspektive}

[79] Ein Aspekt der Formulierung von Rechtssätzen, der in den kantonalen Richtlinien nur selten besprochen wird, ist die Perspektive. Thematisiert wird sie in den Berner Richtlinien, die zwischen sachverhaltsorientierten und personenorientierten Aussagen unterscheiden (BE:4).

[80] Welche dieser beiden Perspektiven verwendet werden soll, hängt laut den Berner Richtlinien davon ab, ob es «eher um Sachverhalte als um Handelnde geht» (BE:4). Dieser Formulierung liegt wohl das Prinzip zugrunde, dass je nach Kontext der eine oder der andere Aspekt einer Aussage stärker gewichtet sein muss. Doch worin besteht dieser Kontext? Ein möglicher Kandidat ist die Tatbestand-Rechtsfolge-Gliederung. Wenn der Tatbestand in erster Linie den Merkmalen einer Person entspricht, bietet sich die personenorientierte Perspektive an. Handelt es sich beim Tatbestand dagegen um einen Umstand, der nicht mit den Merkmalen einer Person zusammenhängt, so bietet sich die sachverhaltsorientierte Perspektive an. Das folgende Beispiel, bei dem der Tatbestand unterstrichen ist, veranschaulicht dieses Prinzip (BE:4):

Vorgesetzte von Betrieben oder Unternehmen, in denen sich im Verkehr mit Sprengmitteln oder pyrotechnischen Gegenständen eine Explosion mit Personen- oder erheblichem Sachschaden ereignet, haben davon unverzüglich der Polizei Kenntnis zu geben.

[81] Dieser Rechtssatz nimmt eine personenorientierte Perspektive ein. Der Tatbestand wird als Merkmal einer Personengruppe (Vorgesetzte) inszeniert, was zu einer seltsam anmutenden Interpretation des Satzes führt: Dass hier eine bestimmte Art von Vorgesetzten verpflichtet wird, und zwar solche, deren Betriebe explodieren. Diese personenorientierte Formulierung ist hier nicht sinnvoll, da es gerade nicht um eine bestimmte Art von Vorgesetzten geht, sondern um eine bestimmte Situation. Deshalb sollte hier, wie es auch die Berner Richtlinien vorschlagen, eine sachverhaltsorientierte Perspektive gewählt werden:

Ereignet sich in Betrieben oder Unternehmen im Verkehr mit Sprengmitteln oder pyrotechnischen Gegenständen eine Explosion mit Personen- oder erheblichem Sachschaden, so ist unverzüglich die Polizei zu benachrichtigen.

[82] Was die sprachliche Umsetzung der Perspektive angeht, bleiben die Richtlinien eher vage und führen lediglich an, dass im Falle der personenorientierten Formulierung «die handelnde bzw. betroffene Person als Subjekt oder wenigstens am Satzanfang erwähnt wird» (BE:4). Etwas spezifischer ist der Gesetzgebungsleitfaden des Bundes, in dem man bis ins Jahre 2019 noch folgende Formulierung fand: «[Die Perspektive hat] insbesondere Auswirkungen auf die Wahl der Verben, auf die Wahl zwischen Aktiv und Passiv, auf die Wahl der Wortstellung im Satz» (Bundesamt für Justiz 2007, Rz. 954).

[83] Dass das Passiv verwendet werden kann, um den Gegenstand einer Handlung gegenüber dem Agens perspektivisch hervorzuheben, wurde bereits in Abschnitt 5.2 diskutiert. Eine ähnliche Wirkung kann eine Veränderung der Wortstellung erzielen, wie folgendes Beispiel zeigt:

Ursprüngliche Formulierung: In besonderen Fällen kann die kantonale Behörde einem Betrieb, der seit mehreren Jahren Lernende mit überdurchschnittlichem Erfolg ausgebildet hat, die Überschreitung der Höchstzahl der Lernenden bewilligen. (Art. 11 
Abs. 5 Verordnung des SBFI über die berufliche Grundbildung Milchpraktikerin/ Milchpraktiker mit eidgenössischem Berufsattest (EBA) ${ }^{23}$ )

Alternative Formulierung: Die kantonale Behörde kann einem Betrieb, der seit mehreren Jahren Lernende mit überdurchschnittlichem Erfolg ausgebildet hat, die Überschreitung der Höchstzahl der Lernenden in besonderen Fällen bewilligen.

[84] In der ursprünglichen Formulierung steht die Präpositionalphrase in besonderen Fällen am Anfang des Satzes, was zu einer perspektivischen Hervorhebung des Tatbestandes gegenüber der Rechtsfolge führt. Alternativ könnte auch das Agens (die kantonale Behörde) perspektiviert werden. Obwohl sich die Norm an die kantonale Behörde richtet, indem sie ihr eine Kompetenz verleiht, ist die ursprüngliche Formulierung sinnvoller. Der Grund dafür ist das Verhältnis der Norm zu den vorangehenden Bestimmungen. Dort wurde die Höchstzahl der Lernenden festgelegt, die ein Betrieb gleichzeitig ausbilden kann. Da Absatz 5 eine Ausnahme zu dieser Regelung darstellt, liegt es nahe, den Tatbestand (im Sinne der Bedingungen für die Ausnahme) perspektivisch hervorzuheben.

[85] Inwiefern die Wahl des Verbs einen Einfluss auf die Perspektive haben kann, soll am folgenden Beispiel aufgezeigt werden:

Ursprüngliche Formulierung: Haben Betriebe mit Prüfeinrichtungen von der Schweizerischen Akkreditierungsstelle [...] eine Akkreditierung erhalten, so berücksichtigt die zuständige Behörde die Ergebnisse des Akkreditierungsverfahrens. (Art. 9 Abs. 4 GLPV ${ }^{24}$ )

[86] Artikel 9 GLPV regelt die Aufgaben und Befugnisse jener Behörden, welche die Betriebe inspizieren, in denen Labortätigkeiten ausgeübt werden (Betriebe mit Prüfeinrichtungen). Bei einer bestimmten Teilmenge der Betriebe - diejenigen, die eine Akkreditierung erhalten haben - muss die inspizierende Behörde zudem die Ergebnisse des Akkreditierungsverfahrens berücksichtigen. Diese Regelung enthält insgesamt drei Akteure: die Behörde, welche die Inspizierung durchführt (zuständige Behörde), der inspizierte Betrieb (Betriebe mit Prüfeinrichtungen) und die Schweizerische Akkreditierungsstelle.

[87] Der primäre Adressat ist auf jeden Fall die zuständige Behörde, denn diese wird zu einer Handlung verpflichtet, falls die Bedingungen erfüllt sind. Wer jedoch dem sekundären Adressaten entspricht, hängt von der Perspektive des Nebensatzes ab, der den Tatbestand ausdrückt. In der oben angeführten, ursprünglichen Formulierung werden durch den Ausdruck eine Akkreditierung erhalten die Betriebe mit Prüfeinrichtungen zum Subjekt gemacht und somit perspektivisch hervorgehoben. Als Alternative käme das Verb akkreditieren in Frage, das die Schweizerische Akkreditierungsstelle perspektivisch hervorheben würde:

Alternative Formulierung: Hat die Schweizerische Akkreditierungsstelle [...] die Betriebe akkreditiert, so berücksichtigt die zuständige Behörde die Ergebnisse des Akkreditierungsverfahrens.

23 Verordnung des SBFI vom 11. Oktober 2019 über die berufliche Grundbildung Milchpraktikerin/Milchpraktiker mit eidgenössischem Berufsattest (EBA) (SR 412.101.220.22).

24 Verordnung vom 18. Mai 2005 über die Gute Laborpraxis (SR 813.112.1). 
[88] Obwohl beide Formulierungen dieselbe Norm zum Ausdruck bringen, unterscheiden sie sich auf der kommunikativen Ebene. Die ursprüngliche Formulierung richtet sich an die Betriebe mit Prüfeinrichtungen und klärt diese darüber auf, wie die zuständige Behörde handeln wird, falls sie eine Akkreditierung erhalten hat. Die alternative Formulierung richtet sich dagegen an die Schweizerische Akkreditierungsstelle und klärt diese darüber auf, dass ihr Handeln zu einer Verpflichtung der zuständigen Behörde führt. Die Wahl des Verbs hat also einen Einfluss auf die Perspektive, und diese wiederum auf die Adressierung eines Rechtssatzes.

\section{Zusammenfassung und Fazit}

[89] Im vorliegenden Artikel wurden die gesetzestechnischen Richtlinien der deutschsprachigen Kantone hinsichtlich ihrer Empfehlungen und Vorgaben zur Gesetzessprache untersucht. Diskutiert wurden drei Themen: die allgemeinen Anforderungen an die Gesetzessprache, die Wortwahl und der Satzbau.

[90] Es herrscht in den Richtlinien grosse Einigkeit darüber, dass die Gesetzessprache einfach, klar und verständlich sein soll. In diesem Zusammenhang werden häufig auch Knappheit und Präzision als wünschenswert genannt. Das Ideal der Knappheit muss dahingehend relativiert werden, dass es sich nicht auf ganze Erlasstexte, sondern auf einzelne Einheiten wie Artikel und Absätze bezieht. Wichtiger als die Länge des gesamten Erlasses ist dessen Aufbau, der das Auffinden der gesuchten Norm erleichtern soll. Zur Verständlichkeit gehört das Ideal der Allgemeinsprachlichkeit, das in Konflikt zur fachlichen Präzision stehen kann. Hier hat sich die Orientierung am Adressatenkreis als massgebliches Kriterium herausgestellt.

[91] Im Abschnitt zur Wortwahl wurden die lexikalische Kohärenz, typische Formulierungsmuster und Legaldefinitionen diskutiert. Die Gesetzessprache ist eine historisch gewachsene Varietät, die über für sie typische Formulierungsmuster verfügt, die teilweise unbewusst verwendet werden. Auf Laiinnen und Laien können diese unverständlich wirken. Allerdings sind auch Vorschläge zur alltagssprachlichen Umformulierung stets mit Vorsicht zu geniessen - die Allgemeinsprachlichkeit darf nicht auf Kosten juristisch relevanter Differenzierungen durchgesetzt werden. Im Zweifelsfalle ist es besser, dass die Leserinnen und Leser etwas nicht verstehen und andere Quellen aufsuchen, als dass sie es falsch verstehen und schlimmstenfalls falsche Entscheidungen treffen.

[92] In Bezug auf den Satzbau haben sich zwei Aspekte als relevant erwiesen: die Informationsdichte und die Struktur der Sätze. Beim Verfassen von Erlasstexten müssen komplexe Sachverhalte so segmentiert werden, dass in jedem Satz idealerweise genau ein Aspekt des Regelungsgegenstandes zum Ausdruck kommt. Dieser Aspekt muss dann möglichst einfach und verständlich formuliert werden. In diesem Zusammenhang wurde auch der Passivsatz diskutiert, dem viele Richtlinien eher kritisch gegenüberstehen. Bei näherer Betrachtung zeigte sich aber, dass er durchaus von Vorteil sein kann.

[93] Zusammenfassend kann gesagt werden, dass die Kantone die generellen Ansprüche an die Gesetzessprache teilen, jedoch in der konkreten Umsetzung variieren können. Nur wenige Richtlinien gehen detailliert auf Letzteres ein. Zudem neigen die Richtlinien dazu, relativ starre Regeln zu formulieren, anstelle von Fall zu Fall zu entscheiden, wann ein Sprachmittel (z. B. ein Passivsatz) sinnvoll ist. Die wissenschaftliche Literatur bietet zwar Hilfe, indem sie die sprachlichen Phänomene der Gesetzessprache reflektiert und konkrete Formulierungsstrategien anbie- 
tet, doch letzten Endes müssen diese Vorschläge immer im jeweiligen Kontext betrachtet und ihr Einsatz abgewogen werden. Nichtsdestotrotz bergen die Unterschiede zwischen den kantonalen Richtlinien eine Chance: Der Blick in die Leitfäden anderer Kantone kann neue Perspektiven auf bekannte Herausforderungen eröffnen und Anstoss zu neuen Überlegungen sein.

MA Lucas Stutz, Doktorand an der Universität Zürich.

\section{Literaturverzeichnis}

Bratschi, RebeKKa (2009): «Frau im Sinne dieser Badeordnung ist auch der Bademeister»: Legaldefinitionen aus redaktioneller Sicht, in: LeGes 20 (2009) 2, 191-213.

Bundesamt für Justiz (2007): Gesetzgebungsleitfaden, 3., nachgeführte Aufl., Bern.

Bundesamt für Justiz (2009): «In der Regel» ergibt in der Regel keine gute Regel, Newsletter für das Forum für Rechtsetzung Nr. 3. Online abrufbar unter: https://www.bj.admin.ch/bj/de/home/staat/legistik/rechtsetzungsforum/news letter.html [03.02.2021].

Bundesamt für Justiz (2019): Gesetzgebungsleitfaden, 4. Aufl., Bern. Online abrufbar unter: https://www.bj.admin.ch/ $\mathrm{bj/de/home/staat/legistik/hauptinstrumente.html} \mathrm{[03.02.2021].}$

BussmanN, Hadumod (2008): Lexikon der Sprachwissenschaft, Stuttgart.

Eichhoff-Cyrus, Karin/Antos, Gerd/Schulz, Rüdiger (2009): Wie denken die Deutschen über die Rechts- und Verwaltungssprache? Eine repräsentative Umfrage der Gesellschaft für deutsche Sprache, Wiesbaden. Online abrufbar unter: https://gfds.de/wp-content/uploads/2014/08/Umfrage_Rechts-_und_Verwaltungssprache.pdf [12.03.2021].

Gesellschaft für deutsche Sprache (1998): Fingerzeige für die Gesetzes- und Amtssprache: Rechtssprache bürgernah, Wiesbaden.

Hagoort, Peter/Indefrey, Peter (2014): The Neurobiology of Language Beyond Single Words, in: Annual Review of Neuroscience 37, 347-362.

Hartmann, Andreas/Parádi, Zsuzsa (2018): Über kurz oder lang: zur angemessenen Kürze in der Formulierung von Rechtsvorschriften, in: Uhlmann, Felix/Höfler, Stefan (Hrsg.), Gute Gesetzessprache als Herausforderung für die Rechtsetzung, Zürich/St. Gallen, 215-238.

Höfler, Stefan (2011): «Ein Satz - eine Aussage»: Multipropositionale Rechtssätze an der Sprache erkennen, in: LeGes 22 (2011) 2, 259-279.

HöFLER, STEFAN (2012): Legislative drafting guidelines: How different are they from controlled language rules for technical writing?, in: Kuhn, Tobias/Fuchs, Norbert E. (Hrsg.), Controlled Natural Language: Third International Workshop CNL 2012, Berlin/Heidelberg, 138-151.

HöFleR, STEFAN (2016): Die Informationsstruktur von Rechtssätzen und ihre Bedeutung für die Gesetzesredaktion, in: LeGes 27 (2016) 2, 225-251.

HöFLeR, STEFAN (2017): Das Legalitätsprinzip in der Gesetzessprache, in: Uhlmann, Felix (Hrsg.), Das Legalitätsprinzip in Verwaltungsrecht und Rechtsetzungslehre, Zürich, 143-171.

Höfler, STEFAN (2018): Gute Gesetzessprache aus dem Blickwinkel der Verwaltung: Die Redaktionskommission der schweizerischen Bundesverwaltung, in: Uhlmann, Felix/Höfler, Stefan (Hrsg.), Gute Gesetzessprache als Herausforderung für die Rechtsetzung, Zürich/St. Gallen, 65-100.

Kindermann, Harald (1979): Ministerielle Richtlinien der Gesetzestechnik: Vergleichende Untersuchung der Regelungen in der Bundesrepublik Deutschland, in Österreich und der Schweiz, Berlin/Heidelberg.

Lötscher, Andreas (1994): Struktur und Adressat: Gesetzesredaktionelle Überlegungen zur Gestaltung von Artikel 9 des Umweltschutzgesetzes (Umweltverträglichkeitsprüfung), in: LeGes 5 (1994) 3, 69-80.

Lötscher, Andreas (2016): Die (Un-)verständlichkeit von Gesetzen: eine Herausforderung für die Gesetzesredaktion, in: ZERL. Online abrufbar unter: http://zerl.uni-koeln.de/loetscher-2016-verstaendlichkeit-von-gesetzen.html [04.03.2021].

Müller, Georg (1976): Richtlinien der Gesetzestechnik in Bund und Kantonen, in: Rödig, Jürgen (Hrsg.), Studien zu einer Theorie der Gesetzgebung, Berlin/Heidelberg, 211-220.

Müller, Georg/Uhlmann, Felix (2013): Elemente einer Rechtssetzungslehre, 3. Aufl., Zürich. 
Nelson, Matthew J./El Karoui, Imen/Giber, Kristof/Yang, Xiaofang/Cohen, Laurent/Koopman, Hilda/Cash, Sydney S./Naccache, Lionel/Hale, John T./Pallier, Christophe/Dehaene, Stanislas (2017): Neurophysiological Dynamics of Phrase-Structure Building During Sentence Processing, in: PNAS 114(18), 3669-3678.

Nussbaumer, Markus (1995): Von den Tücken sprachlicher Erlassoptimierung, in: LeGes 6 (1995) 3, 87-109.

Nussbaumer, Markus (2008): Der Verständlichkeit eine Anwältin! Die Redaktionskommission der schweizerischen Bundesverwaltung und ihre Arbeit an der Gesetzessprache, in: Eichhoff-Cyrus, Karin M./Antos, Gerd (Hrsg.), Verständlichkeit als Bürgerrecht? Die Rechts- und Verwaltungssprache und in der öffentlichen Diskussion, Mannheim u. a., 301-323.

Nussbaumer, Markus (2009): Rhetorisch-stilistische Eigenschaften der Sprache des Rechtswesens, in: Fix, Ulla/Gardt, Andreas/Knape, Joachim (Hrsg.): Rhetorik und Stilistik: Ein internationales Handbuch historischer und systematischer Forschung, 2. Halbband, Berlin/New York, 2132-2150.

Nussbaumer, Markus/Rast Vinzenz/Caussignac Gérard (1995): Verständlichkeit von Gesetzen als Problem der Gesetzgebung, in: LeGes 6 (1995) 1, 87-107.

Ogorek, Regina (2004): «Ich kenne das Reglement nicht, habe es aber immer befolgt!»: Metatheoretische Anmerkungen zur Verständlichkeitsdebatte, in: Lerch, Kent D. (Hrsg.), Recht verstehen: Verständlichkeit, Missverständlichkeit und Unverständlichkeit von Recht, Berlin, 297-305.

OKSAAR, Els (1989): Alltagssprache, Fachsprache, Rechtssprache, in: Zeitschrift für Gesetzgebung 4(3), $210-237$.

Richter, Tobias/Schnotz, Wolfgang (2018): Textverstehen, in: Buch, Susanne R./Rost Detlef H./Sparfeldt, Jörn R. (Hrsg.), Handwörterbuch pädagogische Psychologie, Weinheim, 826-837.

SzUBERT, RAFAL (2013): Bürgernahe Rechts- und Verwaltungssprache? Zur Verständlichkeitsdebatte um eine schicht- und kulturgebundene Fachsprache, in: Grucza, Franciszek/Spiegel, Heinz-Rudi/Schneider-Mizony, Odile/Žmudzki, Jerzy (Hrsg.), Akten des XII. Internationalen Germanistenkongresses Warschau 2010: Vielfalt und Einheit der Germanistik weltweit, Bd. 18: Fachsprachen in Theorie und Praxis, Geschichte des Deutschen als Fremdsprachenunterricht weltweit/Geschichte von DaF weltweit, Theorie und Geschichte der Translationswissenschaft, Frankfurt am Main u. a., $51-56$.

\section{Abkürzungsverzeichnis}

\begin{tabular}{|l|l|}
\hline ZH & Richtlinien der Rechtsetzung (Zürich) \\
\hline BE & Rechtsetzungsrichtlinien des Kantons Bern \\
\hline LU & Richtlinien über die Gesetzestechnik (Luzern) \\
\hline UR & Gesetzestechnische Richtlinien des Kantons Uri \\
\hline SZ & Richtlinien für die Rechtsetzung (Schwyz) \\
\hline OW & Richtlinien über die Erarbeitung und Gestaltung kantonaler Erlasse (Obwalden) \\
\hline GL & Richtlinien für die Rechtsetzung (Glarus) \\
\hline ZG & Leitfaden zur Erlasstechnik des Kantons Zug \\
\hline FR & Gesetzestechnische Richtlinien (Freiburg) \\
\hline SO & Richtlinien zur Gesetzestechnik (Solothurn) \\
\hline BS & $\begin{array}{l}\text { Richtlinien des Regierungsrates des Kantons Basel-Stadt über die formelle Gesetzestechnik und die } \\
\text { geschlechtsneutrale Gesetzessprache }\end{array}$ \\
\hline SH & Richtlinien für die Gestaltung kantonaler Erlasse (Schaffhausen) \\
\hline AR & Gesetzestechnische Richtlinien (Appenzell Ausserrhoden) \\
\hline AI & Richtlinie für die Rechtsetzung (Appenzell Innerrhoden) \\
\hline SG & Rechtsetzungsleitfaden (St.Gallen) \\
\hline GR & Richtlinien für die Rechtsetzung (Graubünden) \\
\hline AG & Richtlinien der Rechtsetzung (Aargau) \\
\hline TG & Richtlinien des Kantons Thurgau für die Gesetzgebung \\
\hline VS & Gesetzestechnische Richtlinien (Wallis) \\
\hline
\end{tabular}

